\title{
Blocking Effects of Mobility and Reservations in Wireless Networks
}

\author{
C. Vargas \\ Ctr. de Elec. y Telecom \\ ITESM \\ Monterrey, MX 68489
}

\author{
M. V. Hegde \\ Dept. of Elec. Engg. \\ Washington University \\ St. Louis, MO 63130
}

\author{
M. Naraghi-Pour \\ Dept. of Elec. and Comp. Engg. \\ Louisiana State University \\ Baton Rouge LA 70803
}

\begin{abstract}
Abstract - We evaluate the effects of mobility and reservations on new call blocking and handoff blocking in multirate wireless networks. The model evaluated uses Fixed Channel Assignment (FCA) with priority for handoffs over new call arrivals by reserving a number of channels for handoff calls in all the cells. The performance measures used are new call blocking and handoff drop probabilities. The methodology used is that of implied costs which we calculate from the the network net revenue which considers the revenue generated by accepting a new call into the network as well as the cost of a handoff drop in any cell. Simulation and numerical results are presented showing the accuracy of the model. We present numerical results showing the effect of reservations on call blocking probability. The implied cost analysis shows that mobility has a significant knock-on effect on the traffic elsewhere in the network and we capture this effect through the net revenue which is sensitive to the level of mobility. We calculate the sum revenue for a given network by maximizing the net revenue using implied costs in a gradient descent algorithm. This analysis indicates that in the case of multiple classes of traffic the call carrying capacity of the network is sensitive to the choice of reservation parameters.
\end{abstract}

\section{Introduction}

Handoff blocking probability is an important criterion in the performance of wireless networks. Typically, on account of customer indignation, the rejection of a handoff is considered to be more undesirable than the rejection of new incoming calls. Essentially three methods are used for the admission of handoffs and new call arrivals. One treats handoff calls and new calls equally for occupancy of the channels, the second reserves channels in each cell to give priority to handoffs and the third sends handoffs to a queue if no channel is available. Performance evaluation algorithms for these strategies have been introduced, for example in [2] for the reservation strategy and the queueing strategy and in [6] for the reservation and no reservation strategy. The trade-offs between new calls and handoff calls are analyzed in [7] where a nonreservation strategy is analyzed for very low and very high mobility of customers. For a treatment of handoff issues and performance comparison of handoff strategies we refer to [5] and for the combination of handoff strategies and channel assignment we refer to [8].
In general, the analysis techniques used for evaluating the performance of wireless networks require fixed point computations to obtain blocking probability and/or handoff drop probability. The use of fixed point computations and the consequent implicitness of the dependence of the blocking probability or the handoff drop probability on the entire network traffic obscure the effects of variables such as exogenous inputs on the performance measures. In this paper we use the concept of implied cost [3], [4] to evaluate these. We assume Fixed Channel Allocation (FCA), (i.e., every cell is assigned a fixed number of channels) with reservation to give priority to handoff calls.

\section{Model for Multirate Wireless Net- works}

Consider an asymmetric cellular network with fixed channel assignment where $\mathcal{N}$ is the set of cells and $N$, the total number of cells. Each cell $i$ has $C_{i}$ channels assigned to it. There are $M$ classes of traffic which share the network resources. Let $b_{m}$ be the number of channels required by traffic of class $m$, $m=1,2, \ldots, M, 0<b_{1} \leq b_{2} \leq \cdots \leq b_{M}$. The new call arrival process of class $m$ to cell $i$ is a Poisson process with mean $\lambda_{i, m}$ independent of other new call arrival processes. The time a call of class $m$ remains in cell $i$, the dwell time, is a random variable with exponential distribution and mean $1 / \mu_{i, m}$ and it is independent of earlier arrival times, call durations and elapsed times of other users. At the end of a dwell time a call may attempt a handoff to an adjacent cell, remain within the cell or leave the network. Let $q_{i j, m}$ be the probability that a call of type $m$ in progress in cell $i$ after completing its dwell time goes to cell $j$, i.e., there is a class $m$ handoff from cell $i$ to cell $j$, let $q_{i T, m}$ be the probability that a call of class $m$ in progress in cell $i$ after completing its dwell time terminates and abandons the network and let $q_{i i, m}$ be the probability that a call of class $m$ in progress in cell $i$ after completing its dwell time remains within cell $i$. If cell $i$ and cell $j$ are not adjacent then $q_{i j, m}=0$ for $m=1, \ldots, M$.

All the cells have a channel reservation parameter, $T_{m}$, for calls of class $m$. The reservation pa- 
rameters are intended to give priority to handoff calls with respect to new calls through a reservation policy as described below. Let $\mathbf{n}=\left(n_{1}, n_{2}, \ldots, n_{M}\right)$ be the current state of cell $i$ where $n_{m}$ is the number of calls of class $m$ present in cell $i$. Define $\mathcal{U}_{i}^{(m)}$ and $\mathcal{B}_{i}^{(m)}$ as the set of unreserved and blocked states for traffic class $m$ in cell $i$, respectively, where $\mathcal{U}_{i}^{(m)}=\left\{\mathbf{n}: T_{m}<\left\lfloor\frac{C_{i}-\sum_{k=1}^{M} n_{k} b_{k}}{b_{m}}\right\rfloor\right\}$ and $\mathcal{B}_{i}^{(m)}=\left\{\mathbf{n}: C_{i}-\sum_{k=1}^{M} n_{k} b_{k}<b_{m}\right\}$, and where $\lfloor x\rfloor$ is the largest integer less than or equal to $x$. Clearly $\mathcal{B}_{i}^{(m)} \subset \mathcal{Q}_{i}^{(m)}$. The reservation policy can be stated as follows. If a new call of class $m$ arrives to cell $i$, it is accepted if the state of cell $i, \mathbf{n}$, is in $\mathcal{U}_{i}^{(m)}$, otherwise, it is blocked. If a handoff call of class $m$ arrives to cell $i$, it is blocked only if $\mathbf{n} \in \mathcal{B}_{i}^{(m)}$.

We consider that occupancy of the cells evolves according to an $M$-dimensional birth-death process independent of other cells. We note however that the transition probabilities of this process depend on the steady state probabilities of the other cells. Let $p_{i}(\mathbf{n})$ be the steady-state probability that cell $i$ is in state n. From $p_{i}(\mathbf{n})$, the new call blocking probability for calls of class $m$ in cell $i, B_{i, m}$, and the handoff drop probability for calls of class $m$ in cell $i, B_{h i, m}$, can be calculated as follows:

$$
B_{i, m}=\sum_{\mathbf{r} \in \mathcal{Q}_{i}^{(m)}} p_{i}(\mathbf{r}), \text { and } B_{h i, m}=\sum_{\mathbf{r} \in \mathcal{B}_{i}^{(m)}} p_{i}(\mathbf{r}) .
$$

In [1] we show that the forced termination probability, $B_{j, m}^{d}$, viz., the probability that a call of class $m$ which originated in cell $j$ is terminated due to a handoff failure during its lifetime can be calculated from these steady-state probabilities as well.

\section{Implied Costs}

Define the net revenue, $W$, as the revenue generated by the traffic which is carried successfully. This revenue consists of two components: the first one is the revenue, $\lambda_{j, m}\left(1-B_{j, m}\right) w_{j, m}$, generated by accepting in each cell $j$ a new call of class $m$ and the second component, $\lambda_{j, m}\left(1-B_{j, m}\right) c_{j, m} B_{j, m}^{d}$, takes into account the cost of a forced termination due to handoff failure of those new calls of class $m$ that have been accepted in cell $j$. Here $w_{j, m}$ is the revenue generated by accepting a call of class $m$ in cell $j$, and $c_{j, m}$ is the cost of a forced termination of a call of class $m$ due to a handoff failure. Hence the net revenue is

$$
W(\underline{\lambda})=\sum_{m=1}^{M} \sum_{j \in \mathcal{N}} \lambda_{j, m}\left(1-B_{j, m}(\underline{\lambda}, \mathbf{p})\right) .
$$

$$
\left\{w_{j, m}-c_{j, m} B_{j, m}^{d}(\underline{\lambda}, \mathbf{p})\right\},
$$

where $\mathbf{p}$ denotes the vector whose components are the steady-state probabilities for each state of all the cells. and $\underline{\lambda}$, the vector of new call arrival rates.

The revenue for cell $i$ and traffic class $m, w_{i, m}$, is proportional to $b_{m}$ and the average holding time of the calls of that class. The average holding time depends on the average number of handoffs the calls undergo before departure from the network and since every time a call is accepted in a cell its duration in that cell is the dwell time with mean $1 / \mu_{i, m}$, the average holding time of a call will be given by the average sum of the dwell times that the call will undergo before its departure from the network. In order to reflect the greater loss of service caused by handoff blocking, $c_{i, m}$ is chosen to be higher than $W_{i, m}$ by a unit.

The fixed point model describes $\mathbf{p}$ as an implicit function of $\underline{\lambda}$. The $B_{j, m}, B_{h j, m}$ and $B_{j, m}^{d}$ are, in turn, functions of $\mathbf{p}$ and thereby implicit functions of $\underline{\lambda}$. Consequently, $W(\underline{\lambda})$ is also an implicit function of $\underline{\lambda}$. We undertake a careful and extensive effort to obtain relations of total and partial derivatives of the new call and handoff blocking probabilities by differentiating the fixed point equations. In particular we calculate the total derivative of the net revenue function with respect to new call arrival rates, $\frac{d W\left(\mathbf{B}, \mathbf{B}_{h}, \underline{\lambda}, \underline{\alpha}\right)}{d \lambda_{k, m}}$. As a result of the analysis we are able to obtain rates of change between different performance measures, between resources in one part of the network and performance in another part, knock-on effects of a change in traffic or resource, all of which are very useful in the design and performance evaluation of these networks.

\section{Numerical Results}

This section presents numerical and simulation results for the model presented in previous sections. We verify the accuracy of the fixed point model, evaluate network revenue for various cases of load and mobility, present examples of how the implied costs can be incorporated into call pricing and obtain the sum revenue for a network with different parameters.

\section{A. Blocking and Revenue Results}

The calculations and simulations were done for the 10-cell network shown in Figure 1 with two classes of traffic. In the figures, $T=[x, y]$ refers to the reservation of $x$ channels for calls of class 1 and $y$ channels for calls of class 2 in all the cells, and $\mu=[x, y]$ refers to a dwell time of $1 / x$ for calls of class 1 and $1 / y$ for calls of class 2 in all the cells. The number of channels needed by each class of traffic are $b_{1}=1$ and $b_{2}=2$. The departure probabilities are $q_{i T, 1}=0.8$ and $q_{i T, 2}$ $=0.85$. These parameters are summarized in Table 
I. The parameters $q_{i j, m}$ are chosen as $\frac{\left(1-q_{i T, 1}\right)}{L}$ where $L$ is the total number of cells the call could move to including the current cell.

In the examples, the channel reservation parameters of the classes were varied and the performance evaluated in terms of the new call blocking probability, $B_{i, m}$, and the handoff drop probability, $B_{h i, m}$, of both classes. The figures shown were obtained by keeping the new call arrival for all the cells and both classes constant except for calls of class 1 in cell 1 since this is one of the cells with the most number of adjacent cells. The total new call arrival rate into a cell for both classes is $20 \%$ of the capacity of that cell.

Figures 2-4 contain graphs of comparison of the numerical results for new call blocking and handoff blocking with the simulations for different channel reservation parameters. In all cases it can be seen that simulation results are extremely close to the numerical results, thereby validating the fixed point model. It can be seen that for both classes of calls, the new call blocking for the case of no reservation performs better than the case with reservation (for handoff drop probability, when channels are reserved, the performance improves). This improvement is due to the priority given to handoffs by reserving channels in each cell. In the case where there is no reservation, Figure 2, the new call blocking and the handoff drop probabilities are the same.

Figure 5 shows the net revenue for the ten cell network for $50 \%$ load, i.e., the new call arrival rate is $50 \%$ of the capacity of that cell. The mobility was varied between low and high: low mobility is characterized by $q_{i T, 1}=0.8$ and $q_{i T, 2}=0.85$ and high mobility is characterized by $q_{i T, 1}=0.5$ and $q_{i T, 2}=0.55$. It can be seen that due to the increase in traffic in the network, because users tend to have longer connection, times the blocking of new and handoff calls will increase and the net revenue will decrease. For the high mobility case, we have less revenue, than that with low mobility. However in the former case reservation can improve the revenue. This can be seen in the figure when we compare the net revenue of the no reservation case, $T=[0,0]$, with that of $T=[1,1]$, or $T=[0,2]$. We can also see in the figure that for high mobility, the case of $T=[2,2]$ will have better revenue than the no reservation case for class 1 new call arrivals above 6 calls per time unit. For the low mobility case, the case of no reservation results in the highest revenue.

In Figure 6, we show the implied cost as a function of new call arrival rate of calls of class one into cell 1. It can be observed that implied cost is a measure of the rate of increase of the net revenue. The larger the implied cost the larger the rate of increase of revenue and the better the blocking level the network is experiencing. The figure also shows that reservation has to be chosen wisely depending on the implied cost obtained: if we have a small value of the implied cost, a connection establishment will be more expensive and it is not a good idea to accept more traffic of that class in that cell. It can be seen that, for high mobility and class 1 new call arrival above five calls per time unit, the implied cost of all the cases of reservation analyzed decrease below the $T=[2,0]$ case, meaning that revenue for this reservation will be better than that of the other cases. In the low mobility case we can see that it will be better to use the reservation $T=[1,1]$ since it will represent an improvement in revenue.

\section{B. Sum Revenue}

Implied costs capture the effect of increases in new call arrivals in one cell on the entire network. As a result, they are useful in optimizing network-wide goals. Define the sum revenue as the maximum sum of new call arrival rates such that the new call blocking probability of each cell is less than or equal to some prespecified maximum blocking probability. The notion of sum revenue is similar to that of sum capacity for circuit switched networks. Sum capacity was introduced and calculated for adaptive routing schemes in [9], and [10]. where implied costs were used to solve a nonlinear constrained optimization algorithm. As an illustration of the use of implied costs in optimizing network-wide goals, we use them to calculate the sum revenue. To this end, we formulate a constrained nonlinear optimization problem with the objective function being the network net revenue and constraints being the new call blocking and handoff blocking probabilities. The independent variables are the new call arrival rates. Let $\underline{\eta}$ and $\underline{\gamma}$ be vectors whose components represent the maximum new call and handoff blocking probabilities, respectively, for each cell and let $\underline{0}$ be the zero vector. Then the optimization problem is:

$$
\begin{array}{r}
\max _{\underline{\lambda}} W\left(\mathbf{B}, \mathbf{B}_{h}, \underline{\lambda}, \underline{\alpha}\right)= \\
\sum_{m=1}^{M} \sum_{i \in \mathcal{N}} w_{i, m} \lambda_{i, m}\left(1-B_{i, m}(\underline{\lambda}, \mathbf{p})\right) \\
-\sum_{m=1}^{M} \sum_{i \in \mathcal{N}} c_{i, m} B_{h i, m}(\underline{\lambda}, \mathbf{p})\left\{\mathcal{I}_{\left\{T_{m}>0\right\}} \alpha_{i, m}(\mathbf{v})\right. \\
\left.+\mathcal{I}_{\left\{T_{m}=0\right\}}\left[\rho_{i, m}(\underline{\lambda}, \mathbf{v})-\lambda_{i, m}\right]\right\}, \\
\text { subject to } \mathbf{B} \leq \underline{\eta}, \mathbf{B}_{h} \leq \underline{\gamma}, \quad \underline{\lambda} \geq \underline{0} .
\end{array}
$$

where $\mathcal{I}($.$) is the indicator function. The solution$ for the above optimization problem gives the maximum revenue that the network can generate for a 
given blocking probability vector. The optimization is achieved by using the implied costs in a gradient descent algorithm that gives the direction in which the vector of new call arrival rates has to be varied to get the desired maximization.

In Figure 7 the sum revenue of the 10-cell network with two classes of customers is shown for several values of channel reservation parameters and for low mobility. The horizontal axis is the new call blocking of class 2 since this is the class with higher bandwidth requirement and its new call blocking is higher than the new call blocking probability of class 1 and the handoff drop probability of both classes. The best performance was obtained for the case of $T=[1,0]$, where there is one channel reserved for handoffs of class 1 . The poorest performance was from the case $T=[0,1]$ and the case $T=[1,1]$, has no significant difference with the $T=[0,1]$ case. The second best case was $T=[0,0]$ with no channels reserved for any class. It can be seen that reservation parameters need to chosen carefully in the multirate case. It can also be concluded that increasing the channel reservation for the class with less bandwidth requirement improves the sum revenue, whereas increasing it for the other class will degrade the performance.

\section{Conclusions}

We described the calculation of implied costs with respect to the new call arrival rates for wireless networks with multiple classes of customers, and show their use for evaluating trade-offs between new call blocking and handoff blocking, and between low mobility and high mobility traffic. Comparison of the sum revenue indicates that the optimization using implied costs results in a significant improvement. This provides evidence that matching capacity distribution to exogenous traffic and mobility can result in significant benefits to the network. The sum revenue in the case of multiple classes of traffic indicates the need for careful choice of reservation parameters.

\section{Acknowledgements}

The second author would like to acknowledge the support of NSF through grant NCR- 9706545.

\section{References}

[1] Vargas C., Shadow Prices for Wireless and Wireline Networks, PhD Thesis, Department of Electrical and Computer Engineering, Louisiana State University, Baton Rouge, Louisiana, July 1996.

[2] Hong, D., and Rappaport S., "Traffic Model and Performance Analysis for Cellular Mobile Radio Telephone Systems with Prioritized and Nonprioritized Handoff Procedures," IEEE Trans. on Vehicular Tech., vol. VT-35, No. 3, August 1986.

[3] Kelly, F.P., "Routing in Circuit-Switched Networks: Optimization, Shadow Prices and Decentralization," Advances in Applied Probability, vol. 20, pp. 112-144, 1988.
[4] Kelly, F.P., "Routing and Capacity Allocation in Networks with Trunk Reservation." Mathematics of Operations Research, vol. 15, pp. 771-792, Nov 1990.

[5] Lin, Y-B., Mohan, S. and Noerpel, A., "Analyzing the Trade-off between Implementation Costs and Performance: PCS Channel Assignment Strategies for Hand-off and Initial Access," IEEE Personal Communications Magazine, Third Quarter, 1994.

[6] McMillan, David, "Traffic Modelling and Analysis for Cellular Mobile Networks," Proceedings of the ITC-13, pp. 627-632, 1991.

[7] Sidi, M., and Starobinski, D., "New Call Blocking versus Handoff Blocking in Cellular Networks," IEEE INFOCOM 96, pp. 35-42, March 1996.

[8] Tekinay, S. and Jabbari, B., "Handover and Channel Assignment in Mobile Cellular Networks," IEEE Communications Magazine, November 1991.

[9] Vargas, C., Hegde, M., Naraghi-Pour, M. and Min, P., "Implied Costs for LLR and ALBA," IEEE/ACM Transactions on Networking, vol. 4, no. 5, pp. 796-807, October 1996.

[10] Vargas, C., Hegde, M., Naraghi-Pour, M. and Min, P., "Shadow Prices for State Dependent Routing," The Twenty-Eighth Annual Conference on Information Sciences and Systems, Princeton University, Princeton, N.J. 08544, March 1994, pp. 243-248.

TABLE I

Parameters Ten-Cell Network, $20 \%$ LoAd, $\left(\lambda_{1,1}\right.$ VAried $)$

\begin{tabular}{|c||c|c|c|c|c|c|c|}
\hline \hline \multicolumn{7}{|c||}{ Simulation Parameters } \\
\hline Cell $i$ & $\lambda_{i, 1}$ & $\lambda_{i, 2}$ & $C_{i}$ & $\mu_{i, 1}$ & $\mu_{i, 2}$ & $q_{i T, 1}$ & $q_{i T, 2}$ \\
\hline \hline 1 & $*$ & 0.1090 & 12 & 1.0 & 0.5 & 0.8 & 0.85 \\
\hline 2 & 2.5454 & 0.1272 & 14 & 1.0 & 0.5 & 0.8 & 0.85 \\
\hline 3 & 3.2727 & 0.1636 & 18 & 1.0 & 0.5 & 0.8 & 0.85 \\
\hline 4 & 4.0000 & 0.2000 & 22 & 1.0 & 0.5 & 0.8 & 0.85 \\
\hline 5 & 3.6363 & 0.1818 & 20 & 1.0 & 0.5 & 0.8 & 0.85 \\
\hline 6 & 2.0000 & 0.1000 & 11 & 1.0 & 0.5 & 0.8 & 0.85 \\
\hline 7 & 2.3636 & 0.1181 & 13 & 1.0 & 0.5 & 0.8 & 0.85 \\
\hline 8 & 2.0000 & 0.1000 & 11 & 1.0 & 0.5 & 0.8 & 0.85 \\
\hline 9 & 3.0909 & 0.1545 & 17 & 1.0 & 0.5 & 0.8 & 0.85 \\
\hline 10 & 2.1818 & 0.1090 & 12 & 1.0 & 0.5 & 0.8 & 0.85 \\
\hline \hline
\end{tabular}

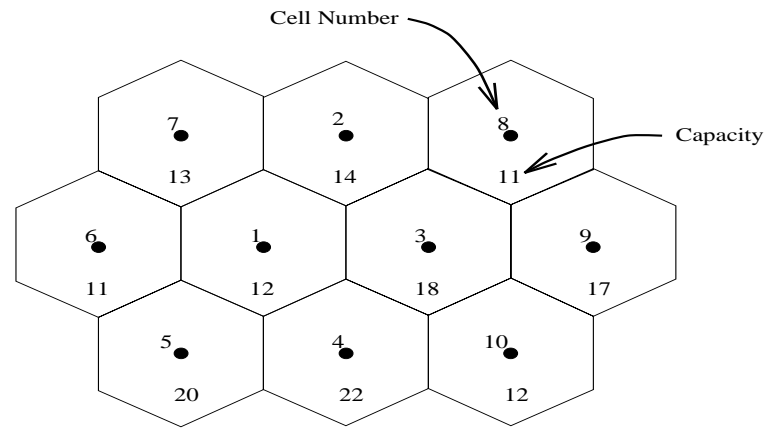

Fig. 1. Ten-Cell Network Used in Examples with Parameters for Single Rate Case 


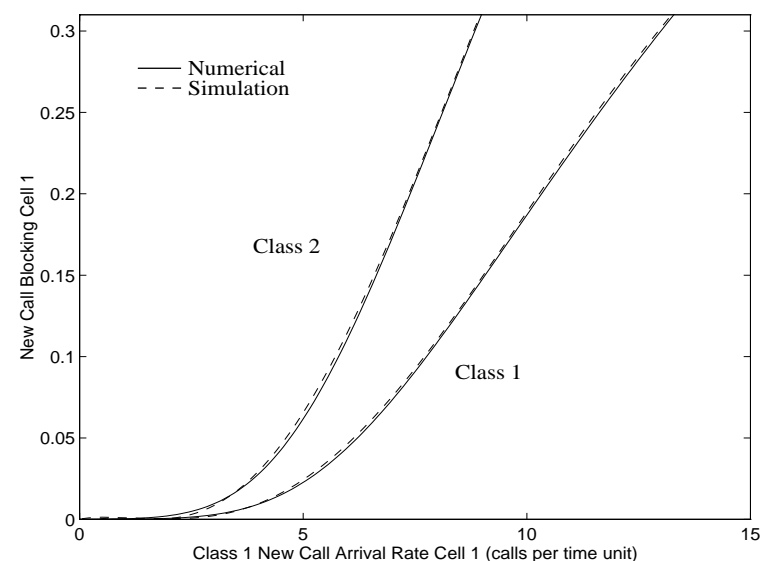

Fig. 2. New call blocking, $T=[0,0], \mu=\left[1, \frac{1}{2}\right], 20 \%$ load

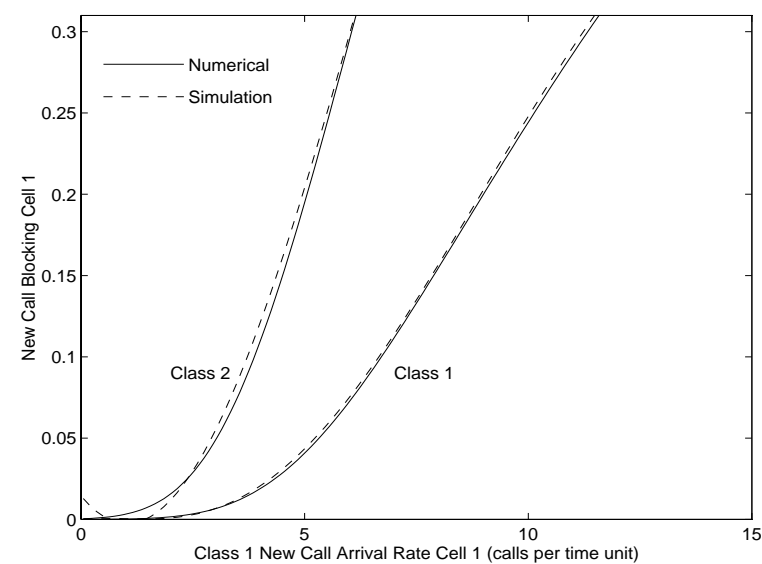

Fig. 3. New call blocking, $T=[1,1], \mu=\left[1, \frac{1}{2}\right], 20 \%$ load

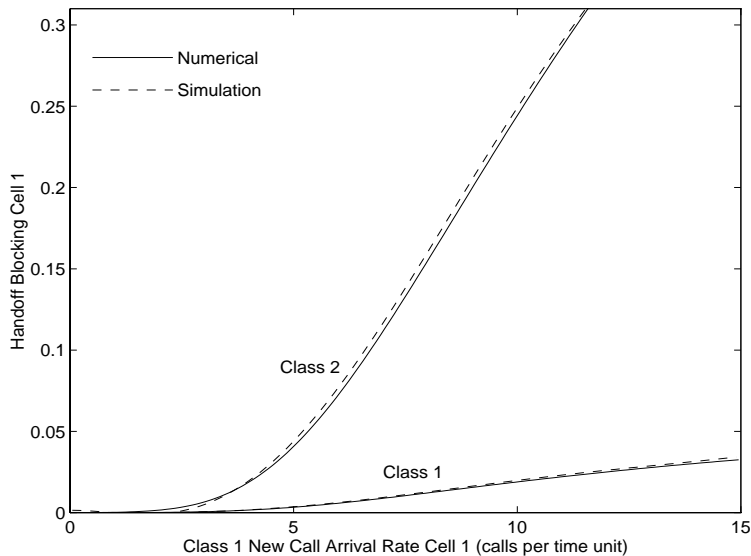

Fig. 4. Handoff blocking probability, $T=[1,1], \mu=\left[1, \frac{1}{2}\right], 20 \%$ load

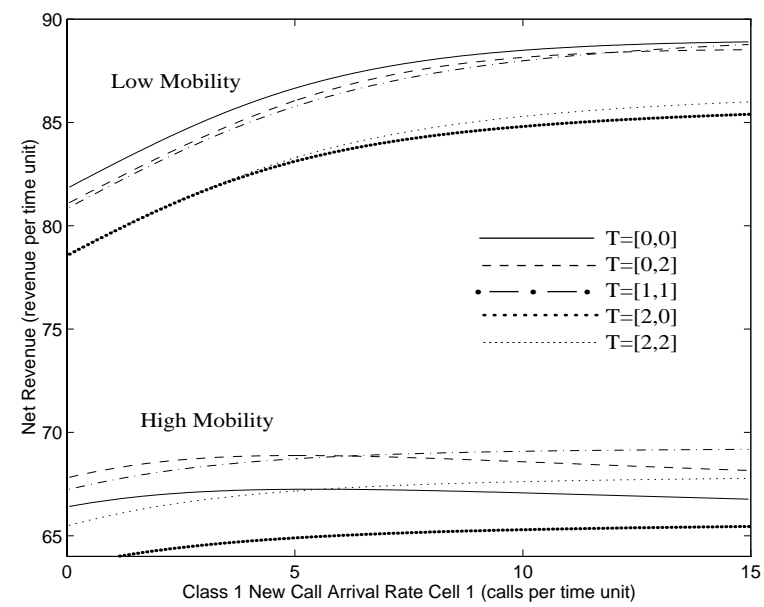

Fig. 5. Net revenue for 10-cell network, $50 \%$ load, $\mu=\left[1, \frac{1}{2}\right]$

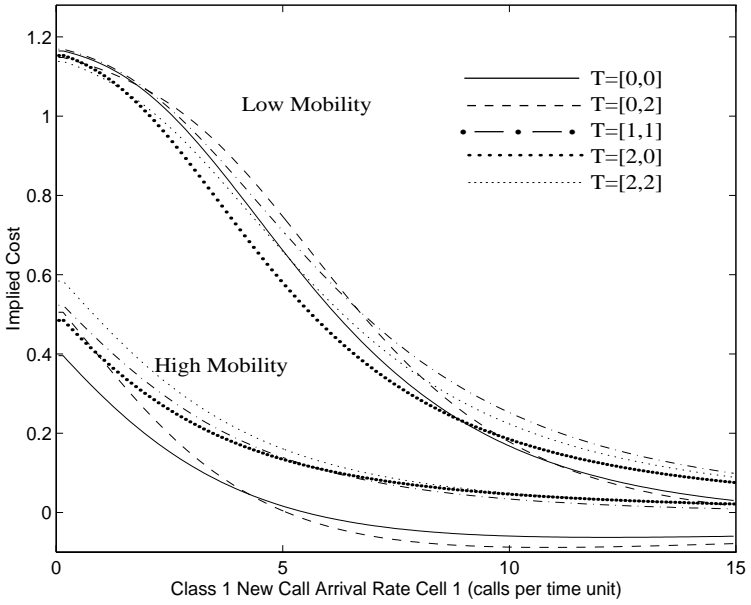

Fig. 6. Implied cost for 10-cell network, $50 \%$ load, $T=[0,0]$, $\mu=\left[1, \frac{1}{2}\right]$

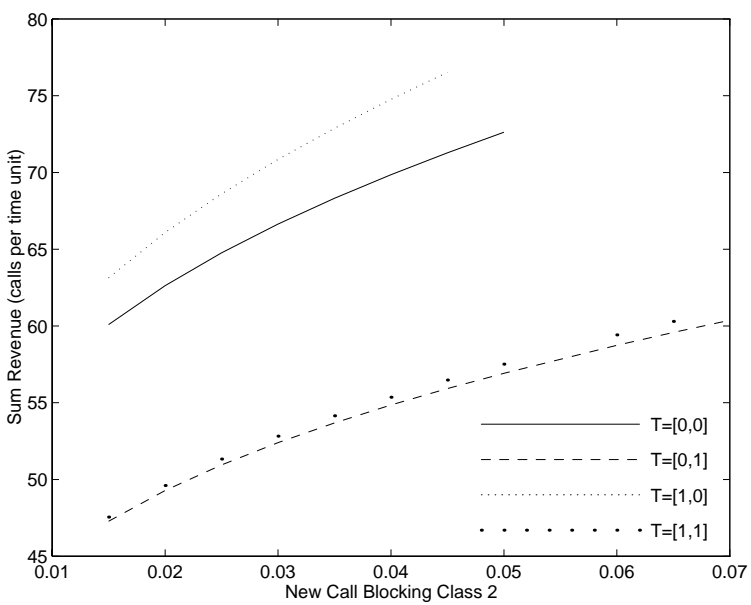

Fig. 7. Sum revenue for the 10-cell network, low mobility 